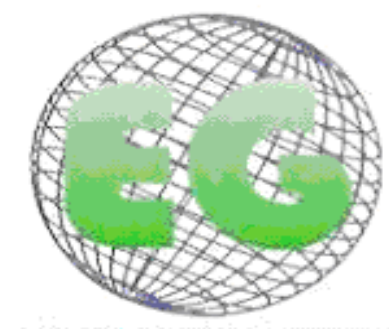

ISSN 1695-6141

$\mathrm{N}^{\circ} 26$

\title{
REVISIONES
}

\section{Hipertensión arterial: principales factores de riesgo modificables en la estrategia salud de la familia}

Hipertensão arterial: principais fatores de risco modificáveis na estratégia saúde da família

\section{*Weschenfelder Magrini, D., **Gue Martini, J.}

\begin{abstract}
*Enfermera. Especialista em Salud Comunitaria, Salud de la Familia y Desarrollo Gerencial de Unidades Básicas del SUS. Miembro del Grupo de Pesquisa EDEN. ** Enfermera. Doctora en Educación. Docente e investigadora del Departamento de Enfermería y del Programa de Pos-Graduación em Enfermería de la UFSC. Brasil.
\end{abstract}

Palabras clave: hipertensión; factores de riesgo; educación para la salud; atención primaria en salud. Palavras-chave: hipertensão; fatores de risco; educação em saúde; atenção primária em saúde.

Keywords: hypertension; risk factors, health education.

\section{RESUMEN}

Este artículo tiene como objetivo el estudio de los principales factores de riesgo de la hipertensión arterial, modificables por la actuación de la Estrategia Salud de la Familia.

La metodología utilizada fue una revisión narrativa de literatura con recogida de datos en la base electrónica SCIELO, con los descriptores: hipertensión, factores de riesgo, equipo multiprofesional y educación en salud. El trabajo ocurrió en el período de diciembre de 2010 a febrero de 2011. Se localizaron 581 artículos sobre HAS, siendo que 11 fueron considerados para el análisis.

Los resultados indican que la educación en salud en la atención básica, especialmente en la Estrategia de Salud de la Familia (ESF) constituye un instrumento de intervención importante, pues conociendo la realidad de la población que atienden, las intervenciones propuestas por el equipo multiprofesional pueden producir resultados positivos.

Se observó la necesidad de valoración de nuevos conceptos de salud-enfermedad y participación del usuario de salud en la elaboración de un plan de intervención, con estímulo al abandono del consumo de tabaco y del uso abusivo de alcohol, reducción de peso entre aquellos con sobrepeso, implementación de actividades físicas, reducción del consumo de sal, aumento del consumo de hortalizas y frutas, además de la disminución en el consumo de alimentos grasos, entre otros, para estimular el auto-cuidado promoviendo una mejora en la calidad de vida de la población.

\section{RESUMO}

Este artigo tem como objetivo o estudo dos principais fatores de risco para a hipertensão arterial. Modificáveis pela atuação da Estratégia Saúde da Família. 
A metodologia utilizada foi a revisão narrativa de literatura com coleta de dados na base eletrônica SCIELO, com os descritores: hipertensão, fatores de risco, equipe multiprofissional e educação em saúde, ocorreu no período de dezembro de 2010 a fevereiro de 2011. Foram localizados 581 artigos sobre HAS, sendo que 11 foram considerados para a análise.

Os resultados apontam que a educação em saúde na atenção Básica, especialmente na Estratégia de Saúde da Família (ESF) constitui-se em um instrumento de intervenção importante, pois conhecendo a realidade da população que assistem, as intervenções propostas pela equipe multiprofissional podem produzir resultados positivos.

Observou-se a necessidade de valorização de novos conceitos de saúde-doença e participação do usuário de saúde na elaboração do seu plano de intervenção, com estímulo à cessação do tabagismo e do uso abusivo de álcool, redução do peso entre aqueles com sobrepeso, implementação de atividades físicas, redução do consumo de sal, aumento do consumo de hortaliças e frutas, além da diminuição de alimentos gordurosos, entre outros, com o intuito de estimular o auto-cuidado promovendo uma melhoria da qualidade de vida da população.

\section{ABSTRACT}

This article aims to study the main risk factors for hypertension, which are modifiable in the Family Health Strategy.

The methodology used was a narrative review of literature to collect data in the electronic SCIELO containing the key words: hypertension, risk factors, multidisciplinary team and health education. Data collection occurred between December 2010 and February 2011. We found 581 articles on hypertension, and 11 were considered for analysis.

The results indicate that health education in primary care, especially in the Family Health Strategy (ESF) is an important intervention tool because knowing the reality of the people who attend, the interventions proposed by the multidisciplinary team can produce positive results.

There is a need for an appreciation of new concepts in health care and health user participation in preparing the plan of action, stimulating smoking cessation and alcohol abuse, weight reduction among the overweight, introduction of physical activity, reduction of salt intake, increased consumption of vegetables and fruits, besides the reduction of fatty foods, among others, in order to stimulate self-care and promote a better quality of life.

\section{INTRODUCCIÓN}

La Hipertensión arterial sistémica (HAS) es considerada al mismo tiempo una enfermedad y un factor de riesgo, presentándose como un gran reto para la salud pública, pues las enfermedades cardiovasculares constituyen la primera causa de muerte en Brasil.

Es definida cuando encontramos valores para la presión arterial sistólica por encima de 140 $\mathrm{mmHg}$ y diastólica arriba de $90 \mathrm{mmHg}$. La presión arterial limítrofe es aquella con valores sistólicos entre $130-139 \mathrm{~mm} \mathrm{Hg}$ y diastólicos entre $85-89 \mathrm{~mm} \mathrm{Hg}$, mientras que consideramos la presión arterial normal sistólica $<130 \mathrm{~mm} \mathrm{Hg}$ y diastólica $<85 \mathrm{~mm} \mathrm{Hg}$. La presión arterial es óptima si la presión arterial sistólica es $<120 \mathrm{mmHg}$ y diastólica $<80 \mathrm{mmHg}$.

Por esa razón la OPAS/OMS busca intensificar y desarrollar estrategias e instrumentos para facilitar el desarrollo de actividades de detección precoz, control permanente y ampliación del nivel de conocimiento de la población mientras patología, factores de riesgo y los impactos causados por la HAS, bien como, las implicaciones que su control y prevención representan para la salud pública. ${ }^{2}$ 
Cada año mueren 7,6 millones de personas en todo el mundo debido a la hipertensión, siendo que $80 \%$ de esas muertes ocurren en países en desarrollo como Brasil, más de la mitad de las víctimas tiene entre 45 y 69 años.

En la Estrategia Salud de la Familia I del municipio de Descanso/SC, verificamos un predominio de 20 casos por 100 personas, o un porcentaje de $32 \%$ considerando personas entre 20 y 60 años de edad. Entre los géneros se constata un predominio de $35 \%$ entre los hombres y $30 \%$ para las mujeres, lo que queda semejante a los datos generales de Santa Catarina.

En Brasil, la hipertensión arterial afecta a más de 30 millones de brasileños, de estos, $36 \%$ de los hombres adultos y $30 \%$ de las mujeres, y es el factor de riesgo más importante para el desarrollo de las enfermedades cardiovasculares, incluyendo el AVC y el infarto del miocardio, que representan las dos mayores causas aisladas de muertes en el país. ${ }^{3}$

La hipertensión arterial es una condición clínica multifactorial caracterizada por elevados y sustentados niveles de presión arterial. Asociada, frecuentemente, a alteraciones funcionales y/o estructurales de los órganos como el corazón, encéfalo, riñones y vasos sanguíneos y las alteraciones metabólicas, con consecuente aumento de riesgo para problemas cardiovasculares fatales y no fatales. ${ }^{3,5,6}$

La hipertensión arterial está presente en todo el mundo, excepto en un pequeño número de individuos que viven en sociedades primitivas, culturalmente aisladas. En las sociedades industrializadas, la presión arterial aumenta constantemente durante las primeras dos décadas. Ya en niños y adolescentes, la hipertensión está asociada al crecimiento y a la maduración. ${ }^{5}$

Otro aspecto que merece atención es el cambio de perfil de la población brasileña en relación al estilo de vida, como hábitos alimentarios, aumento progresivo del predominio de sobrepeso u obesidad, sumado a la baja adhesión a la realización de actividad física, lo que contribuye a este diseño. ${ }^{6}$

Tanto los factores ambientales como los genéticos pueden contribuir a las variaciones regionales y raciales de la presión arterial, así como al predominio de la hipertensión. Estudios indican que sociedades que pasan por cambios de un lugar menos industrializado a uno más industrializado, reflejan en una profunda contribución ambiental para la presión arterial. ${ }^{6}$

Sabemos que la obesidad y el aumento de peso son fuertes e independientes factores de riesgo para la hipertensión; se estima que el $60 \%$ de los hipertensos presentan más del $20 \%$ de sobrepeso. Entre las poblaciones, se observa el predominio de la hipertensión arterial aumentada con relación a la ingestión de la $\mathrm{NaCl}$ e ingestión dietética baja de calcio y potasio lo que puede contribuir para el riesgo de la hipertensión. Mientras los factores ambientales como el consumo de alcohol, estrés psicoemocional y niveles bajos de actividad física también pueden contribuir a la hipertensión. ${ }^{5}$

Con relación a las complicaciones, la hipertensión puede estar asociada a problemas como infarto agudo de miocardio, enfermedades cerebro vasculares, enfermedad renal crónica, vasculopatías periféricas. La Hipertensión Arterial también es llamada asesina silenciosa, debido a que muchos pacientes no presentan ningún síntoma de la enfermedad, resultando difícil establecer un diagnóstico, siendo que, muchas veces, el diagnóstico ocurre por la complicación. ${ }^{7}$ 
Todo ello puede llevar a ausencias en el trabajo, jubilaciones por invalidez, alto coste para la familia y para la salud pública con ingresos, tratamiento medicamentoso y de rehabilitación.

En la búsqueda de control para toda esa problemática, el Ministerio de Salud de Brasil creó en 2002, el programa HIPERDIA que es un Sistema de Registro y Acompañamiento de Hipertensos y Diabéticos de toda la población afectada por esta enfermedad atendida en la Red de Atención Básica. El Sistema permite el acompañamiento de estas personas a través de la salud pública con definición del perfil epidemiológico y planificación de acciones dirigidas para la mejora de su calidad de vida y reducción del coste social.

Dado lo anterior, este estudio buscó evidenciar los principales factores de riesgo para la HAS, divulgados en trabajos científicos, teniendo como problema: ¿Cuáles son los principales factores de riesgo para la Hipertensión Arterial modificables en la Estrategia Salud de la Familia, relatados en la literatura?

\section{DISEÑO METODOLÓGICO}

Este estudio es resultado de una revisión narrativa de literatura dirigida a estudios brasileños que abordan la relación entre la hipertensión arterial y sus factores de riesgo. La colecta de datos ocurrió en el período de diciembre de 2010 hasta febrero de 2011, utilizando los descriptores, hipertensión, factores de riesgo y educación en salud, en libros de propiedad particular del autor y en la base electrónica SCIELO (Scientific Electronic Library Online), teniendo como criterios de inclusión artículos científicos publicados en los últimos cinco años, con acceso a los textos completos.

Tras seleccionar los artículos que atendieron a los criterios de inclusión y a los objetivos del estudio, se hizo un abordaje crítico, identificando cuáles son los principales factores de riesgo relatados que pueden ser modificables por intervención del equipo de la Estrategia Salud de la Familia, principalmente, a través de prácticas educativas que pueden ser estimuladas en atención multiprofesional individual y colectiva.

El presente trabajo permitió localizar un total de 581 artículos. Tras la lectura de los resúmenes se incluyeron en el estudio 11 artículos que atendieron a los criterios de inclusión, publicados en el período de 2006 y 2010.

Tabla 1 - Artículos utilizados en el presente estudio sobre Hipertensión Arterial y sus factores de riesgo

\begin{tabular}{|c|c|c|}
\hline TÍTULO DEL ARTÍCULO & $\begin{array}{l}\text { PERIÓDICO-REVISTA } \\
\text { PUBLICACIÓN }\end{array}$ & AÑO \\
\hline Hipertensión, Diagnóstico y clasificación & Revista Brasilera de Hipertensión & 2010 \\
\hline Palabra del Presidente & Revista Brasilera de Hipertensión & 2010 \\
\hline $\begin{array}{l}\text { Hipertensión Arterial y Algunos Factores de } \\
\text { Riesgo en una Capital Brasileña }\end{array}$ & $\begin{array}{l}\text { Revista de la Sociedad Brasilera } \\
\text { de Cardiología }\end{array}$ & 2007 \\
\hline $\begin{array}{l}\text { Educación en Salud en el Enfrentamiento de la } \\
\text { Hipertensión Arterial: Una nueva Óptica Para un } \\
\text { viejo Problema }\end{array}$ & $\begin{array}{l}\text { Revista Educación en Salud en el } \\
\text { Enfrentamiento de la Hipertensión } \\
\text { Arterial }\end{array}$ & 2007 \\
\hline $\begin{array}{l}\text { Prevalencia y factores de riesgo para } \\
\text { hipertensión en una población urbana brasileña }\end{array}$ & Archivos Brasileros de Cardiología & 2010 \\
\hline $\begin{array}{l}\text { Conceptuación, Epidemiologia y Prevención } \\
\text { Primaria }\end{array}$ & Revista Brasilera de Hipertensión & 2010 \\
\hline
\end{tabular}




\begin{tabular}{|l|l|l|}
\hline $\begin{array}{l}\text { Hipertensión arterial y su correlación con } \\
\text { algunos factores de riesgo en ciudad brasileña } \\
\text { de pequeño porte }\end{array}$ & $\begin{array}{l}\text { Archivos } \\
\text { Cardiológicos }\end{array}$ & 2010 \\
\hline $\begin{array}{l}\text { Circunferencia de la cintura e índice de masa } \\
\text { corporal como predictores de la hipertensión } \\
\text { arterial }\end{array}$ & Archivos Brasileros de Cardiología & 2006 \\
\hline $\begin{array}{l}\text { Frecuencia de factores de riesgo cardiovascular } \\
\text { en voluntarios participantes de evento de } \\
\text { educación en salud }\end{array}$ & $\begin{array}{l}\text { Revista de la Asociación Médica } \\
\text { Brasileña }\end{array}$ & 2009 \\
\hline $\begin{array}{l}\text { Comportamiento en salud entre ancianos } \\
\text { hipertensos }\end{array}$ & Revista de Salud Pública & 2009 \\
\hline $\begin{array}{l}\text { Frecuencia de Hipertensión Arterial y Factores } \\
\text { de Riesgo Asociados: Brasil 2006 }\end{array}$ & Revista de Salud Pública & 2009 \\
\hline
\end{tabular}

Fuente: Elaborado por la autora

\section{RESULTADOS Y DISCUSIONES}

Los datos encontrados permiten afirmar que tras la implantación del Programa Salud de la Familia, en 1994, hubo un aumento significativo en las publicaciones y estudios, creemos que esto ha ocurrido como consecuencia de la necesidad que los profesionales de salud sintieron en buscar metodologías pertinentes para la actuación en sus realidades locales.

En el estudio realizado por Cipullo (2010) fue registrado un menor predominio de HAS en las regiones Norte $(18,9 \%)$ y Medio Oeste $(19,4 \%)$ y una prevalencia mayor en las regiones Sudeste $(22,8 \%)$ y Sur $(20,9 \%)$. El mismo estudio señaló también que la frecuencia de la hipertensión aumenta con la edad, disminuye con la escolaridad, es mayor entre negros y viudos y menor entre los solteros, aumentando entre los individuos con sobrepeso, diabetes, dislipidemias y con problemas cardiovasculares. ${ }^{8}$

En los artículos analizados, encontramos como factores de riesgo modificables por la intervención de los profesionales de la ESF la edad, factores genéticos, género y etnia, factores socioeconómicos, exceso de peso y obesidad, sedentarismo, ingestión de alcohol, tabaco y hábitos alimentares. Presentamos, a continuación, los destacados de cada factor de riesgo relatado en la literatura encontrada.

\section{Edad}

Al investigar la ocurrencia de HAS en relación a la edad, se observa un aumento progresivo de hipertensión en relación a la edad, llegando hasta el $70 \%$ entre los individuos con más de 70 años, siendo que el promedio estudiado fue de 25,3\% con edad entre 18 y 93 años. Cipullo et al (2010) y Costa et al (2009) ${ }^{8,14}$

Ávila et al (2010) relata la existencia de una relación directa de la Presión Arterial con la edad, siendo la prevalencia de HAS superior al $60 \%$ en individuos con más de 65 años. ${ }^{9}$

\section{Factores Genéticos}

Según Ávila et al (2010) existe una correlación entre los factores genéticos y la hipertensión arterial, todavía no existen variantes genéticos que puedan determinar el riesgo individual de desarrollo de la hipertensión arterial. ${ }^{9}$ 
Kuschnir y Mendonça (2007) relatan que en los últimos años está siendo estudiada cuál es la asociación de la desnutrición intrauterina con patologías degenerativas, tales como la hipertensión, enfermedades coronarias, entre otras. Se cree que la hipertensión puede ser determinada por alteraciones en sistemas biológicos, originarios a partir de la combinación de genes, contribuyendo al aumento en los niveles tensionales de la presión arterial. ${ }^{16}$

\section{Género y etnia}

En relación al género y etnia la prevalencia de la hipertensión arterial es semejante entre hombres y mujeres, pero más elevada en los hombres hasta los 50 años, cambiando a partir de la quinta década. En relación a la etnia, la hipertensión arterial es dos veces más predominante en individuos negros, según investigación realizada por Ávila et al (2010) y Ferreira et al $(2009)^{9,15}$

\section{Factores socioeconómicos}

Las diferencias socioeconómicas tienen un papel importante en la vida de las personas pudiendo determinar sus condiciones de salud, pues aquellas con mejores condiciones tienen mayor acceso a las informaciones, mejor entendimiento de la condición clínica y mayor adherencia al tratamiento. Hay tasas más altas de enfermedades cardiovasculares en grupos con nivel socioeconómico más bajo. Cipullo et al $(2010)^{8}$

La baja escolaridad está asociada a las mayores tasas de enfermedades crónicas no transmisibles, en especial a la hipertensión arterial. NASCENTE $(2010)^{10}$

\section{Exceso de peso y obesidad}

Estudios de Ávila et al (2010) relatan que el exceso de peso se asocia con mayor predominio a la hipertensión arterial desde edades jóvenes, y que en la vida adulta, incluso entre individuos no sedentarios, un incremento de $2,4 \mathrm{~kg} / \mathrm{m}^{2}$ en el índice de masa corporal resulta en mayor riesgo de desarrollar la hipertensión. ${ }^{9}$

En estudios de Costa et al (2009) y Nascente (2010) se puede ver que la obesidad lleva a un envejecimiento no exitoso. El riesgo de morir presenta una relación con el índice de masa corporal, así personas con exceso de peso tienen mayor probabilidad de desarrollar varias patologías como hipertensión, diabetes tipo 2 , entre otras. ${ }^{14,10}$

Peixoto et al (2006) sugieren que aproximadamente $70 \%$ de los casos nuevos de hipertensión arterial pueden ser atribuidos a la obesidad o al aumento de peso. ${ }^{11}$

Los datos sobre aumento de sobrepeso y obesidad, refuerzan la necesidad de implementación de medidas objetivas para su combate a través de acciones en la Estrategia Salud de la Familia, disminuyendo la morbidad y mortalidad de las enfermedades cardiovasculares. Nascente $(2010)^{10}$

La fuerte relación entre la obesidad y la hipertensión arterial indica la urgencia de medidas educativas capaces de actuar sobre los factores de riesgo que pueden determinar el predominio de la hipertensión arterial. (JARDIM,2007) ${ }^{6}$ 


\section{Sedentarismo}

En cuanto al sedentarismo, Ávila et al (2010) refieren que la actividad física reduce la incidencia de HAS en individuos pre hipertensos además de reducir la mortalidad y los riesgos de desarrollar enfermedades cardiovasculares. ${ }^{9}$

Las actividades físicas reducen la mortalidad por problemas cardiovasculares, independientemente de la presión arterial y de otros factores de riesgo, existiendo fuertes evidencias de que la actividad física disminuye la presión sanguínea, prediciendo un envejecimiento saludable. Costa et al (2009) ${ }^{14}$

\section{Ingestión de alcohol}

Para Ávila et al (2010) la ingestión prolongada de alcohol puede, además de aumentar la presión sanguínea, aumentar la mortalidad cardiovascular en general. ${ }^{9}$

\section{Tabaquismo}

Costa et al (2009) refiere que el tabaquismo es la mayor causa de mortalidad por problemas cardiovasculares del mundo, aunque el cese del hábito de fumar no disminuya los niveles de presión arterial, el abandono es la medida más efectiva para la reducción de los riesgos de problemas cardiovasculares. ${ }^{14}$

\section{Hábitos alimentarios}

Jardim (2007) y Ávila et al (2010) relatan que el perfil de la población brasileña cambió en relación a los hábitos alimentarios y de vida, pues esta se expone de forma cada vez más intensa a los riesgos cardiovasculares. El cambio en las cantidades de alimentos ingeridos y la composición de la dieta, sumada a la baja frecuencia de la actividad física, provocó alteraciones significativas en el peso corporal y en la distribución de la obesidad, aumentando su prevalencia en la población. ${ }^{6,9}$

El consumo de sal excede los límites máximos recomendados para la ingestión en todos los países, en todos los extractos de renta, sabemos que la restricción de sal acompañada de hábitos alimentarios saludables contribuye a la reducción de la presión arterial, pudiendo llevar a la reducción de la medicina anti-hipertensiva. Costa et al (2009) y Ávila et al $(2010)^{14,9}$

Los hábitos alimentarios influyen directamente en la morbimortalidad cardiovascular. El estudio realizado por Costa et al (2009) señala que dietas ricas en frutas y hortalizas y productos con poca grasa disminuyen el riesgo cardiovascular, contribuyen a la reducción del peso y reducción de la presión arterial. ${ }^{14}$

Coltro et al (2009) creen que las medidas educativas que estimulen los cambios en el estilo de vida y hábitos alimentarios de la población todavía son insuficientes, sospechándose que la población no tenga amplio acceso a las informaciones sobre la prevención primaria de diversas enfermedades. ${ }^{12}$ 
En la Estrategia Salud de la Familia I del municipio de Descanso, los profesionales tienen conocimiento de las condiciones alimentarias de las personas y deben actuar en la educación para la salud discutiendo con la población estrategias para evitar el aumento de peso, previniendo y reduciendo la hipertensión arterial y sus complicaciones cardiovasculares.

Para Toledo (2007), la Estrategia Salud de la Familia se caracteriza como un modelo clavado en el desarrollo de acciones preventivas y de promoción a la salud de los individuos, familias y comunidades. Siendo que la promoción de la salud es entendida como un proceso de capacitación de comunidad para promover su mejora en la calidad de vida y salud, incluyendo su participación en este proceso. ${ }^{7}$

Coltro et al (2009) relatan que el predominio de factores de riesgos cardiovasculares modificables en la población evaluada indica que existe un grado no despreciable de desinformación sobre prevención de estos factores, sugiriendo la necesidad constante de medidas de salud pública que promuevan educación y prevención primaria, intensificándose esas medidas en individuos ancianos y con sobrepeso.

La hipertensión arterial no ocurre de forma aislada, pues la mayoría de los hipertensos presenta otros factores de riesgo cardiovasculares. Muchos de esos factores de riesgo son modificables, así las recomendaciones para el cambio del estilo de vida son de extrema importancia tanto para la prevención como para el control de la hipertensión arterial (Costa et al,2009) ${ }^{14}$

En ese estudio podemos percibir que los principales factores de riesgo para la hipertensión arterial, como la obesidad, sedentarismo, hábitos alimentarios inadecuados, tabaquismo, abuso de alcohol pueden influir de forma aislada como también aumentan su potencial cuando sumados a más de un factor de riesgo para la misma enfermedad.

La implementación de estilos de vida que afectan de forma favorable a la población lo mismo puede prevenir el riesgo de desarrollar que puede contribuir al tratamiento de la hipertensión arterial. Así se hace necesaria una atención especial a cada factor de riesgo modificable, como el control del peso y la obesidad, porque sabemos que una modesta pérdida de peso puede disminuir los niveles de la presión arterial.

Para mantener una buena salud cardiovascular y tener calidad de vida, todo persona debe realizar por lo menos tres veces por semana, al menos treinta minutos, alguna actividad física, desde que tenga condiciones de realizarla, porque además de facilitar la pérdida de peso, la actividad física ayuda al control de la presión arterial.

La manutención de hábitos alimentarios saludables con ingestión de frutas y verduras, la reducción de frituras y grasas, además de la moderación en el consumo de bebidas alcohólicas y tabaco pueden traer reducción efectiva en la presión arterial.

Como la Hipertensión Arterial es una enfermedad multifactorial necesita un abordaje por equipo multiprofesional. En el municipio de Descanso están siendo desarrolladas muchas acciones por los equipos de la Estrategia Salud de la Familia a través de intervenciones individuales y actividades colectivas en las comunidades, con charlas, entrega de medicación anti-hipertensiva, control de los niveles de presión arterial y peso, además de la elaboración e implementación de nuevos proyectos como el Proyecto Descanso con Más Salud que está siendo desarrollado en la Estrategia Salud de la Familia I. El objetivo de este proyecto es proporcionar la práctica de actividad física y recreación para las personas, como 
una forma de ayudar en el tratamiento y principalmente en el control de enfermedades, además de otros proyectos, con apoyo de equipo multiprofesional de soporte con educadores físicos, fisioterapeutas, psicólogos, cardiólogo, entre otros, que actúan siempre promoviendo el auto cuidado, la mejora en la calidad de vida de la población y un envejecimiento saludable.

\section{CONSIDERACIONES FINALES}

Creemos que la educación en salud en la Atención Básica, en especial en la Estrategia Salud de la Familia, es uno de los principales elementos de promoción de la salud, pues además de que los profesionales conozcan los factores de riesgo para diversas enfermedades, aquí nos referimos a la Hipertensión arterial, saben cuáles son los hábitos de vida de aquella población, siendo así más fácil implementar una intervención positiva.

Para eso, además de la necesidad de cambio de paradigmas biomédicos y de la valoración de nuevos conceptos sobre el proceso salud/enfermedad, es necesario que los usuarios de salud sean coproductores de un proceso educativo para los cambios de hábitos, contribuyendo para que disminuya la frecuencia de varios problemas, incluyendo la hipertensión arterial, mejorando la calidad de vida de la población y garantizando un envejecimiento saludable.

Las actividades educativas dirigidas a la población deben tener por objetivo prevenir la hipertensión arterial; para aquellos que ya padecen la hipertensión arterial las actividades deben ser enfocadas a la reducción de los niveles de presión arterial, control de otros factores de riesgo cardiovasculares y la reducción del uso de medicamentos antihipertensivos. Las estrategias recomendadas deben orientarse al cese del tabaquismo y del uso abusivo de alcohol, reducción del peso entre aquellos con sobrepeso, implementación de actividades físicas, reducción del consumo de sal, aumento del consumo de hortalizas y frutas, además de la disminución de alimentos grasos, estímulo al auto cuidado y la promoción de una vida saludable.

Creemos que este trabajo puede servir principalmente para que los profesionales de la salud piensen en cómo se da la relación profesional/paciente y cuál es la forma de repaso de informaciones para una población que necesita de informaciones para el mantenimiento de una calidad de vida saludable, ya que se hace mucho y a veces con poco resultado.

\section{REFERENCIAS}

(1) Pierin, Angela M.G. et al. Revista Brasileira de Hipertensão: VI Diretrizes Brasileiras de Hipertensão, Diagnóstico e classificação. Rio de Janeiro: v.17, n.1, p.11-17, 2010.

(2) Organização Mundial de Saúde. Disponible en: <http://new.paho.org>. Acceso en: 16 oct. 2010.

(3) Malachias, Marcus V. B. Revista Brasileira de Hipertensão: VI Diretrizes Brasileiras de Hipertensão, Palavra do Presidente. Rio de Janeiro: v.17, n.1, p.2-3, 2010.

(4) DATASUS. Disponible en: <http://siab.datasus.gov.br>. Acceso en: 11 enero 2011.

(5) Fauci, Anthony S, et al. Harrison Medicina Interna. Rio de Janeiro: 17ª edición, volumen 2., McGraw-Hill, 2008.pg. 1549-1562.

(6) Jardim, Paulo César B. Veiga. Hipertensão Arterial e Alguns Fatores de Risco em uma Capital Brasileira. Revista da Sociedade Brasileira de Cardiologia. v.88 n.4, p.452-457. 2007. 
(7) Toledo, Melina Mafra. Educação em Saúde no Enfrentamento da Hipertensão Arterial: Uma nova Ótica Para um velho Problema. Revista Educação em Saúde no Enfrentamento da Hipertensão Arterial. v.16 n.2, p.233-228, Abr- jun. 2007.

(8) Cipullo, José Paulo, et al. Prevalência e fatores de risco para hipertensão em uma população urbana brasileira. Arquivos Brasileiros de Cardiologia. vol.94. n4, São Paulo, abr.2010.

(9) Ávila, Adriana, et al. Revista Brasileira de Hipertensão, VI Diretrizes Brasileiras de Hipertensão, Conceituação, Epidemiologia e Prevenção Primária, Rio de Janeiro, v.17, n.1, p.7-10, 2010.

(10) Nascente, Flávia Miquetichuc Nogueira. Hipertensão arterial e sua correlação com alguns fatores de risco em cidade brasileira de pequeno porte. Arquivos Brasileiros de Cardiológicos, Epub 27 - Ago. 2010.

(11) Peixoto, et al. Circunferência da cintura e índice de massa corporal como preditores da hipertensão arterial.Arquivos Brasileiros de Cardiologia. vol.87 n.4 São Paulo oct. 2006.

(12) Coltro et al. Frequência de fatores de risco cardiovascular em voluntários participantes de evento de educação em saúde. Revista da Associação Médica Brasileira, vol.55 n.5. São Paulo 2009.

(13)

Ministerio

De

la

Salud.

http://portal.saude.gov.br/portal/se/datasus/area.cfm?id_area=807. Acceso en: 1 de feb. 2011.

(14) Costa, Maria F. F. de L, et al. Comportamento em saúde entre idosos hipertensos. Revista de Saúde Pública vol.43 supl.2 São Paulo, no 2009.

(15) Ferreira, Sandra R. G, et al. Frequência de Hipertensão Arterial e Fatores de Risco Associados: Brasil 2006. Revista de Saúde Pública vol.43 supl 2, São Paulo, Nov.2009.

(16) Kuschnir, Maria C. C., Mendonça, Gulnar A. S. Fatores de risco associados à hipertensão arterial em adolescentes. Jornal de Pediatria v.83 n.4 Porto Alegre, Jul-ago.2007.

ISSN 1695-6141

๑ COPYRIGHT Servicio de Publicaciones - Universidad de Murcia 